\title{
Throughput Analysis of Full-Duplex Communication Cognitive Radio Network
}

\author{
Mir Muhammad Lodro ${ }^{1} \cdot$ Steeve Greedy $^{2}$ - Naeem A. Mahoto ${ }^{3}$. \\ Mukhtiar A. Unar ${ }^{3}$
}

Published online: 31 July 2017

(C) The Author(s) 2017. This article is an open access publication

\begin{abstract}
In this paper we deal with the throughput of full-duplex cognitive communication radio which exploits unused band of primary user (PU) network. Classical cognitive radio uses half-duplex communication spectrum sensing to perform spectrum sensing and data transmission at different time intervals. It's well-established fact that in half-duplex communication cognitive radio spectrum sensing time increases at low SNR which gives rise to lesser data transmission time for secondary user (SU) and hence results in less throughput for SU. It's useful idea to do spectrum sensing and data transmission at the same time with two different antennas co-located on the SU transceiver. This shall not only guarantee high probability of detection of PU but also increased data transmission which means more throughput for SU. However, simultaneous sensing and data transmission has inherent problem of self-interference. One of the possible solution is to use polarisation discrimination in which sensing and data transmission antennas must use different polarisation. This is feasible if there is prior information about the polarisation of the signals emitted by the PUs. It shall be of special interest to assess throughput using analytical expressions for probability of detection $P_{D}$, probability of false alarm $P_{F A}$ at various values of SNR for time-slotted cognitive radio which uses half-duplex spectrum sensing and nontime-slotted cognitive radio which uses full-duplex communication cognitive radio.
\end{abstract}

Keywords Full-duplex cognitive radio - Spectrum sensing · Sensing-throughput . TV white space

Mir Muhammad Lodro

mir.lodro@iba-suk.edu.pk; mir.lodro@nottingham.ac.uk

1 The Department of Electrical Engineering, Sukkur Institute of Business Administration, Sukkur 65200, Sindh, Pakistan

2 The University of Nottingham, Nottingham, UK

3 Mehran UET Jamshoro, Jamshoro, Pakistan 


\section{Introduction}

Recently because of the influx of smartphone and due to digital dividend there has been increased trend for application that demand high data rates. Due to digital dividend and shift in user pattern a lot of under-utilised spectrum is observed typically in VHF and UHF band of electromagnetic spectrum and such sparsely used bands are also known as TV white spaces (TVWS). Cognitive radio (CR) has been considered as a powerful technique to increase the spectral efficiency by enabling unlicensed secondary users to access underutilized spectrum opportunistically. Federal Communication Commission (FCC) in USA and Office Communication (Ofcom) in UK have legalised the usage of TVWS in VHF and UHF part of the spectrum. In USA non-contiguous TVWS are located in frequency range of 54-698 MHz and portion of frequency ranging from $470 \mathrm{MHz}$ to 790 $\mathrm{MHz}$ exists in Europe. There are some new standards which involve cognitive radio principle few to mention are wireless metropolitan area network (WMAN) IEEE 802.22 for broadband applications in remote areas, wireless local area network (WLAN) standard known as IEEE 802.11af which uses master and slave architecture and notion of white space database, IEEE $802.15 .4 \mathrm{~m}$ an low data rate short range wireless personal area network (WPAN) system with cognitive radio and a host of other network types shall experience cognitive radio based transformation. Performance of cognitive radio is assessed by the type of detector it uses and the number of sub-bands it can harness and relinquish if the incumbent user reactivates and reclaims its licensed band. A number of detectors has been considered for the implementation in CR transceivers for example energy detector [1, 2], matched filter detector [3, 4], Eigen-value based detector [5, 6], detectors involving cyclostationarity [7] and wavelet-based detection [8] etc.

\section{Background Study}

The problem of reactivation failure is associated with the non-time-slotted cognitive radio networks (NTS-CRN) where the PU and SU traffic is not synchronised i.e. PU randomly leaves and reclaims its licensed channel. In time-slotted CRN PU and SU traffic is synchronised i.e. SU transmits after its senses that there is no incumbent user present on that band. In non-time-slotted CRN PU may sense the busy channel since it can't distinguish between PUs traffic and SU traffic and thus goes into back off stage and retransmits with persistent carrier sense multiple access (p-CSMA) which might result in collision and ultimately affect the PU throughput if the SU doesn't sense the PU reactivation and leave licensed PU sub-channel. For time-slotted CRN we use half-duplex spectrum sensing scheme and medium access control (MAC) protocol. Similarly, for non-time-slotted CRN a novel full-duplex spectrum sensing scheme and a novel cognitive medium access control (CMAC) protocol can be used.

Liang et al. [9] developed seminal expression on sensing-throughput of cognitive radio where he found optimal sensing period which achieves maximum throughput and reference [10] proposes full-duplex spectrum sensing algorithms for non-time-slotted full-duplex cognitive radio. Because of ubiquitous nature of OFDM based communication systems work in [11] focuses on sensing-throughput tradeoff of the OFDM based cognitive radio and the probability of false alarm expression in Nakagami-m and Rician fading channels. Reference [12] studies the interference caused by SU due to missed detection of PU and the extent to which PU is affected in terms of capacity-outage performance under Rayleigh and 
Nakagami fading channels and suggests beacon placement at transmitter and receiver. Novel and computationally efficient detection algorithm for OFDM based PUs is discussed in [13] where the performance of various detectors in AWGN and Rayleigh fading channels is also assessed. Multi-antenna sensing and Generalized Likelihood Ratio (GLR) detector is investigated when channel gain, noise variance and PU signal values are unknown [14]. Ghassemi et al. [15] proposes peak-to-average-power ratio (PAPR) reduction in non-contiguous OFDM (NC-OFDM) using selected mapping (SLM) sequence which results in out-of-band (OOB) reduction and improved PAPR and bit error rate (BER) for NC-OFDM signals, whereas PAPR reduction in OFDM based CR using clockwise subcarrier activation is presented in [16] and a novel signal cancellation method is proposed in [17] for joint PAPR reduction and sidelobe suppression in NC-OFDM based CR network. Work in [18] deals with cooperative spectrum sensing with energy-detector over multipath fading and shadowing with different fusion strategies and using single and multiple cognitive relays. Work in [19] calculates energy efficiency versus optimal sensing time for CR network. Reference [20] presents the cooperative wideband spectrum sensing over fading channels and models the aliasing effects of sub-Nyquist sampling. Yunfei Chen [21] gave an energy detector with enhanced performance for random signals in Gaussian noise. Energy efficient cognitive radio for joint spectrum sensing and data transmission which uses co-located layer of relays with amplify-and-forward strategy to relay data from source to destination has been proposed in [22].

The remainder of the paper is structured as follows: Sect. 3 explains Full-duplex communication for non-time-slotted cognitive radio and the control model for NTS-CR and TS-CR. It also presents the Full-duplex spectrum sensing algorithm for FDC-CR with which it declares the absence and presence of PU. Section 1 defines the system model for NTS-CR and presents the probability of detection and probability of false alarm used for receiver operating characteristic curve (ROC) to see the detector performance and the throughput for secondary user. Following this Sect. 5 compares the throughput of halfduplex and full-duplex communication cognitive radio. Finally, Sect. 6 contains the numerical results on probability of detection, probability of false alarm, probability of missed detection and throughput of the HDC and FDC cognitive radio.

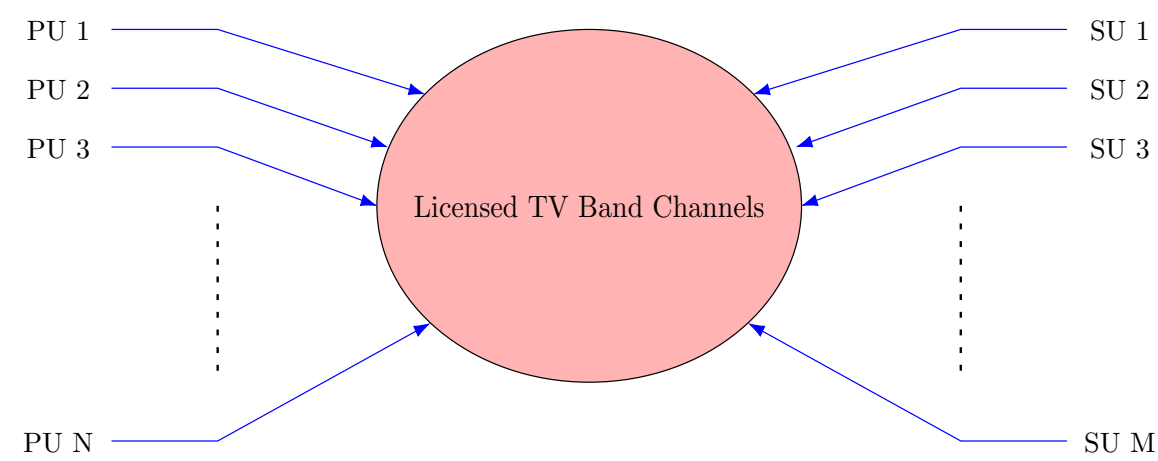

-N PUs have high priority to access the licensed TV band
-M PUs maximise their throughput given that PUs are protected

Fig. 1 Multi-channel cognitive radio network model 

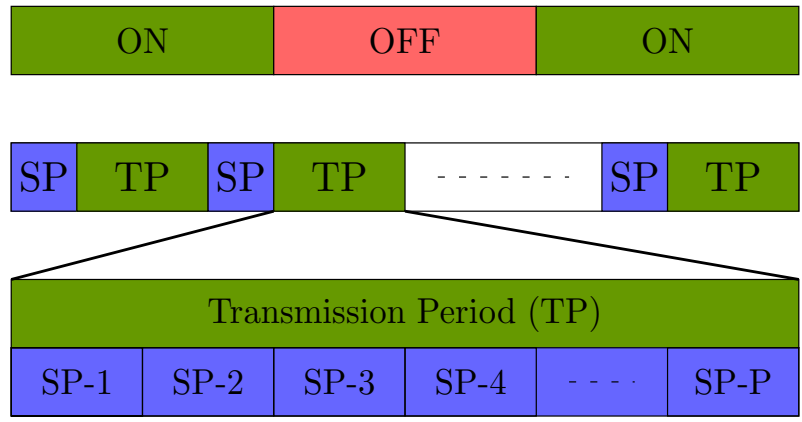

Fig. 2 Control model for NTS-CR

\section{Full-Duplex Communication Non-time Slotted CR}

In NTS-CR using FDC-SS we use two antennas for sensing and transmission simultaneously in contrast to TS-CR in which one antenna is used for periodic sensing and transmission. In TS-CR MAC frame is divided into sensing-time and data transmission time. It is a proven fact that PU protection is guaranteed at increased sensing time which reduces the data transmission time, thereby affecting the throughput of SU. In the following section we discuss problem of reactivation failure for NTS-CR network (NTS-CRN).

\subsection{NTS-CRN Control Model}

In TS-CRN PU traffic and SU traffic is synchronous i.e. SU scans for the availability of under-utilised channel in its fixed sensing duration and quits after it performs data transmission in a fixed transmission time. Figure 2 shows control model of the NTS-CRN where we can see the PU traffic and SU traffic is asynchronous because PU and SU don't quit and reclaim the channel within fixed time interval. In NTS-CRN we divide transmission period of the SU into $P$ sensing periods for sensing the channel with different antenna.

\subsection{Reactivation Failure of NTS-CRN}

In NTS-CRN since the PU and SU arrive and quite channel randomly therefore collision may take place if the PU wants to reclaim licensed channel when SU is transmitting. In this scenario PU can't recognise the traffic of peer PU or SU i.e. whether licensed channel is occupied by peer PU or SU, therefore PU goes in back-off stage and transmits with p-CSMA. If the licensed channel is occupied by SU then collision takes place and PU goes into back-off stage and senses the channel but if the network time is expired PU discards the traffic. PU's such failure to reclaim its licensed channel is known as reactivation failure. This problem of reactivation failure can be solved with FDC-SS algorithm as shown in Algorithm 1 in which SU senses for the arrival of PU during transmission phase. 


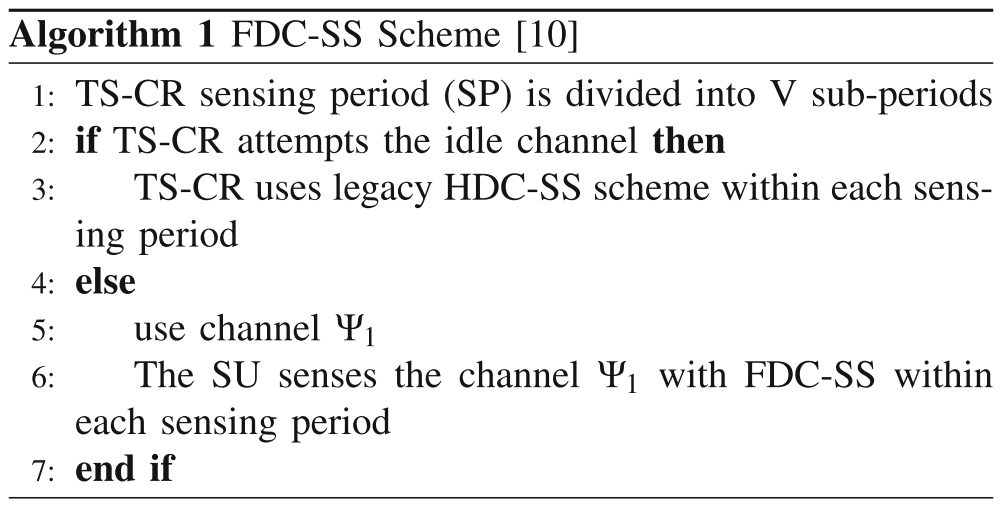

\subsection{FDC-SS Scheme}

Figure 4 shows non-slotted multi-channel cognitive radio network model where PUs use their L licensed sub-channels and M SUs opportunistically contend for the PU licensed sub-channels.

\section{FDC-SS System Model}

We can write the sampled output of CR employing energy-detector as follows:

$$
y(n)=\sqrt{k} h s(n)+w(n) n=0,1,2, \ldots, N-1
$$

where $h$ is channel gain between PU and SU and has Rayleigh distribution. $s(n)$ is sampled PU signal, $w(n)$ is sampled AWGN with zero mean and variance $\sigma_{w}^{2}$ i.e. $\mathcal{N} \sim\left(0, \sigma_{w}^{2}\right)$ and $k$ is self-interference introduced because of the simultaneous sensing and transmission that takes place in NTS-CR. Where $k=0$ means maximum self-interference is introduced and no self-interference for $k=1$. The test statistics using FDC-SS at the output of the energy detector of NTS-CR can be given as in Eq. 2.

$$
T_{N}(y)= \begin{cases}\frac{1}{N}\left(\sum_{n=0}^{u}|\sqrt{k} h s(n)+w(n)|^{2}+\sum_{n=u+1}^{N}|w(n)|^{2}\right), & \text { if } \mathcal{H}_{10} \\ \frac{1}{N}\left(\sum_{n=0}^{v}|w(n)|^{2}+\sum_{n=v+1}^{N}|\sqrt{k} h s(n)+w(n)|^{2}\right), & \text { if } \mathcal{H}_{01}\end{cases}
$$

Where hypothesis $\mathcal{H}_{10}$ means PU is active for first $u$ samples and then becomes inactive for rest of the samples $n=u+1$ to $N$. and hypothesis $\mathcal{H}_{01}$ means the PU was inactive for the first $v$ samples and then became active for rest of the samples $n=v+1$ to $N$. $N$ is total number of samples for entire sensing period. Here we consider PU signal $s(n)$ and noise $w(n)$ as circularly symmetric complex Gaussian (CSCG) which represents signals with rich-ISI such as OFDM. For large number of samples $N$ we can invoke central limit theorem (CLT) under hypothesis $\mathcal{H}_{10}$ and the PDF of the test statistics $T_{N} y, p_{10}(x)$ can be approximated by Gaussian distribution. Thus the $P_{D}$ and $P_{F A}$ of NTS-CR respectively can be given mathematically as in Eqs. 3 and 4 respectively. 


$$
\begin{aligned}
P_{F A}(\xi, N, u, k) & =\operatorname{Pr} \cdot\left(T_{N}(y)>\xi \mid \mathcal{H}_{10}\right)=\int_{\xi}^{\infty} p_{10}(x) d x \\
& =Q\left(\frac{\frac{\xi}{\sigma_{w}^{2}}-\frac{u}{N} k \gamma_{p s}-1}{\sqrt{\frac{u}{N^{2}}\left(k \gamma_{p s}+1\right)^{2}+\frac{N-u}{N^{2}}}}\right) \\
P_{D}(\xi, N, v, k) & =\operatorname{Pr} \cdot\left(T_{N}(y)>\xi \mid \mathcal{H}_{01}\right)=\int_{\xi}^{\infty} p_{01}(x) d x \\
& =Q\left(\frac{\frac{\xi}{\sigma_{u}^{2}}-\frac{N-v}{N} k \gamma_{p s}-1}{\sqrt{\frac{N-v}{N^{2}}\left(k \gamma_{p s}+1\right)^{2}+\frac{v}{N^{2}}}}\right)
\end{aligned}
$$

where $\xi$ is the threshold of the energy detector employed at the receiver. Now we can set $u=0, v=0$ and $k=1$ in above equations and we can obtain $P_{F A}(\xi, N, u, k)$ and $P_{D}(\xi, N, v, k)$ for TS-CR as follows:

$$
\begin{gathered}
P_{F A}(\xi, N, u, k)=Q\left(\left(\frac{\xi}{\sigma_{w}^{2}}-1\right) \sqrt{N}\right) \\
P_{D}(\xi, N, v, k)=\left(\left(\frac{\xi}{\sigma_{w}^{2}}-\gamma_{p s}-1\right) \frac{\sqrt{N}}{\gamma_{p s}+1}\right)
\end{gathered}
$$

From above two equations we can see that the $P_{F A}$ and $P_{D}$ for TS-CR are special case of NTS-CR. For a given $\bar{P}_{D}, P_{F A}$ relates to $P_{D}$ and for a given $\bar{P}_{F A}, P_{D}$ relates to $P_{F A}$ respectively as follows [9]:

$$
\begin{gathered}
P_{F A}=Q\left(\sqrt{2 \gamma+1} Q^{-1}\left(\bar{P}_{D}\right)+\sqrt{\tau f_{s}} \gamma\right) \\
P_{D}=Q\left(\frac{1}{\sqrt{2 \gamma+1}}\left(Q^{-1}\left(\bar{P}_{F A}\right)-\sqrt{\tau f_{s}} \gamma\right)\right)
\end{gathered}
$$

Similarly, for given $\left(\bar{P}_{D}, \bar{P}_{F A}\right)$, the minimum number of samples are given by:

$$
N_{\text {min }}=\frac{1}{2}\left[Q^{-1}\left(\bar{P}_{F A}\right)-Q^{-1}\left(\bar{P}_{D}\right) \sqrt{2 \gamma+1}\right]^{2}
$$

\section{HDC-SS and FDC-SS Sensing-Throughput Comparison}

HDC-SS involves MAC frame with duration $T$ which consists of sensing time $\tau$ and data transmission time $(T-\tau)$. Whereas FDC-SS involves sensing and data transmission time equal to entire frame duration $T$ with collocated antennas used for spectrum sensing and data transmission respectively. There are two scenarios for which SU can operate in PU's licensed band: 
Scenario I When PU is not present and no false alarm is generated by SU Scenario II When PU is present but it's not detected by SU.

In both scenarios the achievable throughputs of the SU link are $\left(\frac{T-\tau}{T}\right) \log _{2}\left(1+P_{s} / N_{0}\right)$ and $\left(\frac{T-\tau}{T}\right) \log _{2}\left(1+P_{s} / P_{o}+N_{0}\right)$ respectively. Where $P_{s}=$ received power of SU, $P_{p}=$ PU power as an interference to SU and $N_{0}$ is the noise power. The probability of occurrence of scenario I and scenario II are $\left(1-P_{F A}(\xi, N, u, k)\right) P\left(\mathcal{H}_{0}\right)$ and $\left(1-P_{D}(\xi, N, v, k)\right) P\left(\mathcal{H}_{1}\right)$. Let us say if:

$$
\begin{aligned}
R_{0}(\xi, N, u, k)= & \left(\frac{T-\tau}{T}\right) \log _{2}\left(1+S N R_{s}\right) \\
& \times\left(1-P_{F A}(\xi, N, u, k)\right) P\left(\mathcal{H}_{0}\right) \\
R_{1}(\xi, N, v, k)= & \left(\frac{T-\tau}{T}\right) \log _{2}\left(1+\frac{S N R_{s}}{S N R_{p}}\right) \\
& \times\left(1-P_{D}(\xi, N, v, k)\right) P\left(\mathcal{H}_{1}\right)
\end{aligned}
$$

Then the average throughput of the $\mathrm{SU}$ is:

$$
R=R_{0}(\xi, N, u, k)+R_{1}(\xi, N, v, k)
$$

Now for HDC-SS longer the sensing time $\tau$, shorter is the data transmission time $T-\tau$ which results in less throughput for SU. Similarly for FDC-SS let us assume that there is no self-interference and SU operates over entire frame duration $T$. Following the same steps we can derive average throughout for NTS-CR with HDC-SS. Now average throughput of the TS-CR and NTS-CR with HDC-SS and FDC-SS respectively is as follows:

$$
\begin{aligned}
& R_{0}(\xi, N, u, k)+R_{1}(\xi, N, v, k)<R_{0}^{F}(\xi, N, u, k)+R_{1}^{F}(\xi, N, v, k) \\
& \left(\frac{T-\tau}{T}\right)\left\{\log _{2}\left(1+S N R_{s}\right)\left(1-P_{F A}(\xi, N, u, k)\right) P\left(\mathcal{H}_{0}\right)\right. \\
& \left.\quad+\log _{2}\left(1+\frac{S N R_{s}}{1+S N R_{p}}\right)\left(1-P_{D}(\xi, N, v, k)\right) P\left(\mathcal{H}_{1}\right)\right\} \\
& \quad<\log _{2}\left(1+S N R_{s}\right)\left(1-P_{F A}(\xi, N, u, k)\right) P\left(\mathcal{H}_{0}\right) \\
& \quad+\log _{2}\left(1+\frac{S N R_{s}}{1+S N R_{p}}\right)\left(1-P_{D}(\xi, N, v, k)\right) P\left(\mathcal{H}_{1}\right)
\end{aligned}
$$

From Eq. (13) it is clear that the average throughput of NTS-CR employing FDC-SS is greater than the throughput of the TS-CR employing HDC-SS. 


\section{Numerical Results and Discussion}

Figure 3 shows the probability of detection versus energy-detection threshold for the for NTS-CR without self-interference i.e. $k=1$. We can see that the probability of detection decreases as the threshold increases. It also decreases with the increase in number of samples for which PU is initially absent i.e from $v=500$ to $v=1500$. Figure 4 shows the probability of missed detection versus threshold for NTS-CR with no self-interference. We can see the probability of missed detection increases with the increase in threshold $\epsilon$. It also increases with the increase in number of samples assigned to PU for which initially remains inactive.

Figure 5 shows the probability of detection of TS-CR and NTS-CR with HDC-SS and FDC-SS spectrum sensing scheme. Here we consider the effect of self-interference and compare the probability of detection with self-interference and without self-interference. We can see the $P_{D}$ for TS-CR outperforms all the cases of NTS-CR because the PU exists for some samples. Moreover, the $P_{D}$ decreases as the energy-detector threshold increases, it also decreases for NTS-CR with the self-interference and without self-interference. However, the $P_{D}$ without self-interference is higher than the $P_{D}$ with self-interference, but the performance gap is very small. Similarly, Fig. 6 shows the probability of missed detection which increases with the increase in energy-detection threshold. It also increases with the increase in number of initial sample values for which PU remains inactive. The probability of missed detection also increases with the increase in self-interference $k$. There is small performance gap between probability of missed detection with self-interference and without self-interference, but there is significant difference in probability of missed detection for TS-CR and the probability of missed detection when the number of initial samples for which PU is inactive increase to $v=1500$. For example at energy-detection threshold of $\xi=1$ the probability of missed detection is 0.2 which increases to $P_{M D}$ value

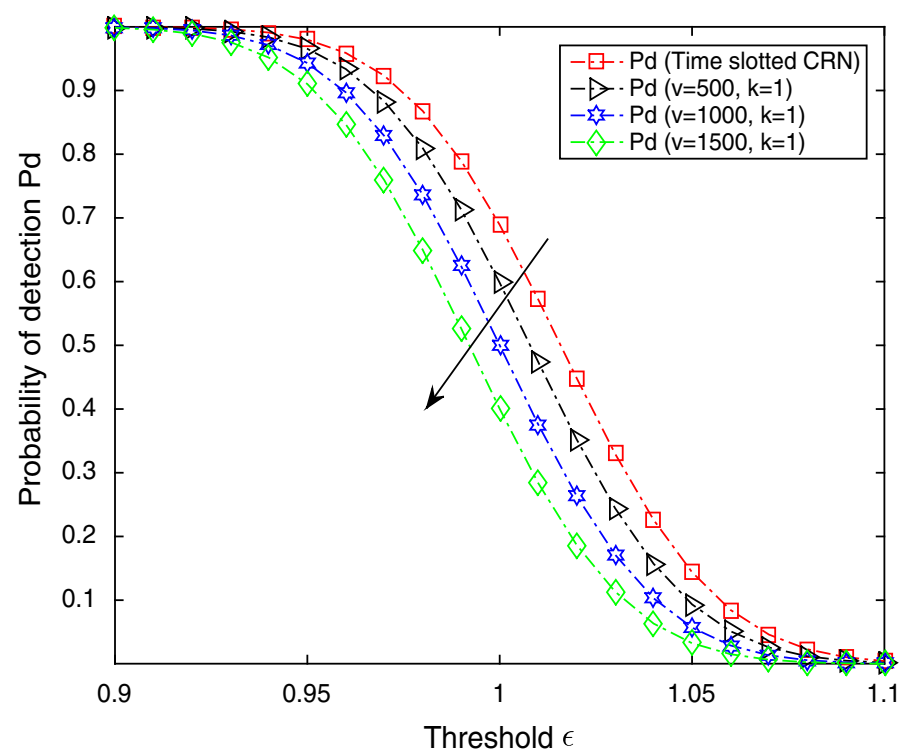

Fig. 3 Probability of detection versus threshold of TS-CR with HDC-SS and NTS-CR with FDC-SS without self-interference 


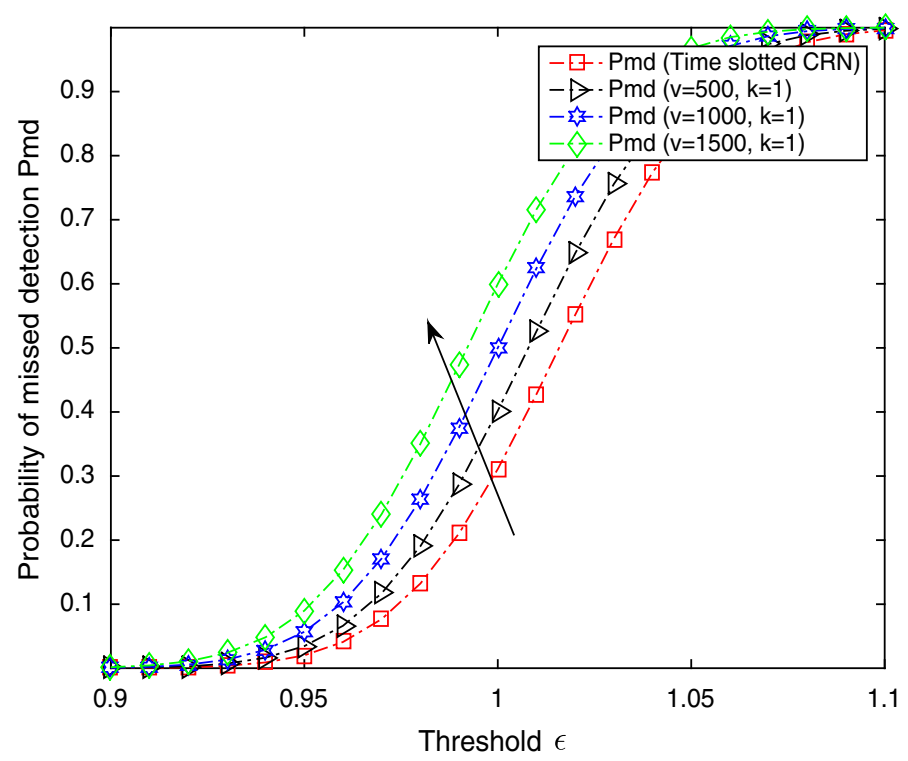

Fig. 4 Probability of missed detection versus threshold of TC-CR with HDC-SS and NTS-CR with FDC-SS without self-interference

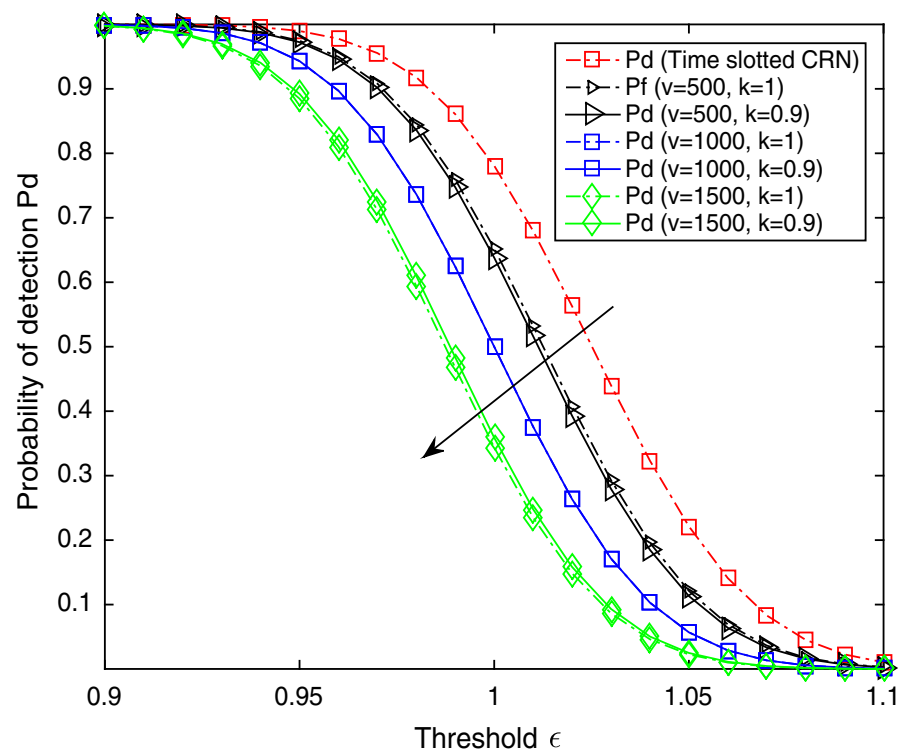

Fig. 5 Probability of detection versus threshold of TS-CR with HDC-SS and NTS-CR with FDC-CR with and without interference

of 0.55 for PU which remains inactive for initial sample values of $v=1500$. We summarise different values of $P_{D}, P_{M D}$ and $P_{F A}$ in the following Table 1 . Note that we calculate the $P_{F A}$ when he PU is active initially for $u$ samples and then leave the channel for rest of the sample values (Tables 1,2 ). 


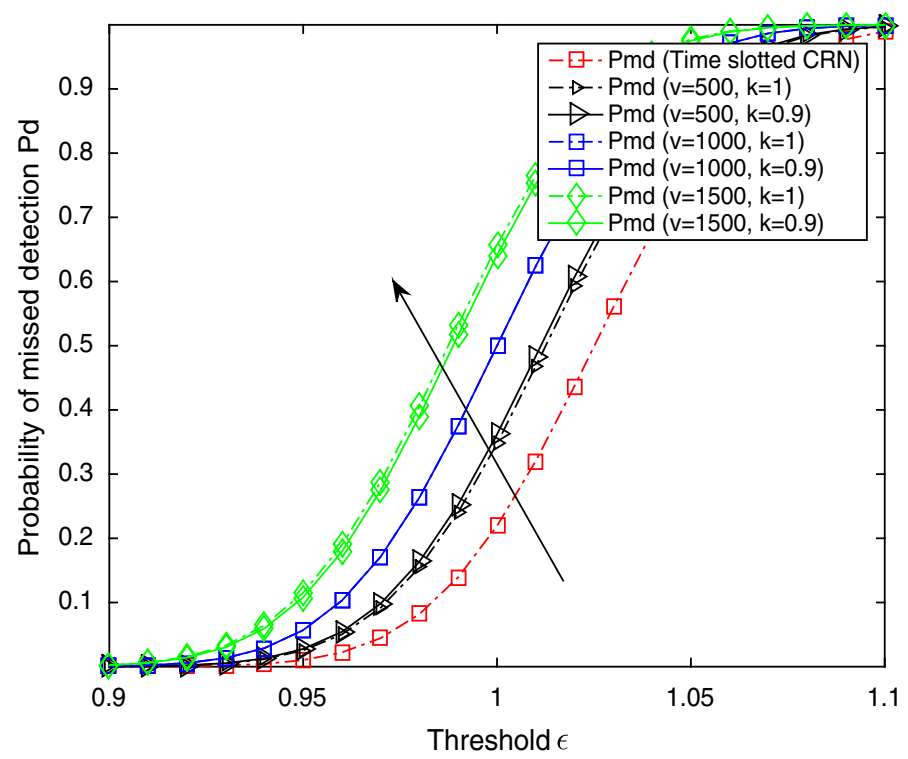

Fig. 6 Probability of missed detection versus threshold of TS-CR with HDC-SS and NTS-CR with FDC-SS with and without interference

Table $1 P_{D}, P_{M D}$ and $P_{F A}$ values for non-time slotted CRN with self-interference and without selfinterference

\begin{tabular}{llllllr}
\hline Threshold $(\xi)$ & \# of samples $(v)$ & $\begin{array}{l}\text { Self- } \\
\text { interference }(k)\end{array}$ & $P_{D}$ & $P_{M D}$ & $u$ & $P_{F A}$ \\
\hline 1 & 500 & 1 & 0.68 & 0.32 & 500 & 0.70 \\
1 & 500 & 0.9 & 0.65 & 0.35 & 500 & 0.68 \\
1 & 1500 & 1 & 0.28 & 0.72 & 1500 & 0.92 \\
1 & 1500 & 0.9 & 0.25 & 0.75 & 1500 & 0.90 \\
\hline
\end{tabular}

Table 2 Throughput of TS-CR with HDC-SS and NTS-CR with FDC-SS at SNR values of $-2 \mathrm{~dB}$

\begin{tabular}{llll}
\hline $\begin{array}{l}\text { Sensing time } \\
(\tau)(\mathrm{ms})\end{array}$ & $\begin{array}{l}\text { Throughput }(b / s / H z): \text { TS- } \\
\text { CR with HDC-SS }\end{array}$ & $\begin{array}{l}\text { Throughput }(b / s / H z): \text { NTS- } \\
\text { CR with FDC-SS }\end{array}$ & $\begin{array}{l}\text { Percent increase } \\
\text { in throughput }(\%)\end{array}$ \\
\hline 6 & 3.6367 & 3.8688 & 5.9 \\
10 & 3.5899 & 3.9888 & 10 \\
12 & 3.5145 & 3.9937 & 11.9 \\
16 & 3.3557 & 3.9949 & 16 \\
\hline
\end{tabular}

Figures 7 and 8 on probability of false alarm follows our intuition developed after our earlier discussions on $P_{D}$ and $P_{M D}$ with and without self-interference for TS-CR and NTS$\mathrm{CR}$. We can see the probability of false alarm of TS-CR is less when the PU is initially active for $u=500$ to $u=1500$ samples and later subsides and leaves the channel empty. This is also true for the $P_{F A}$ values with self-interference and without self-interference. For 


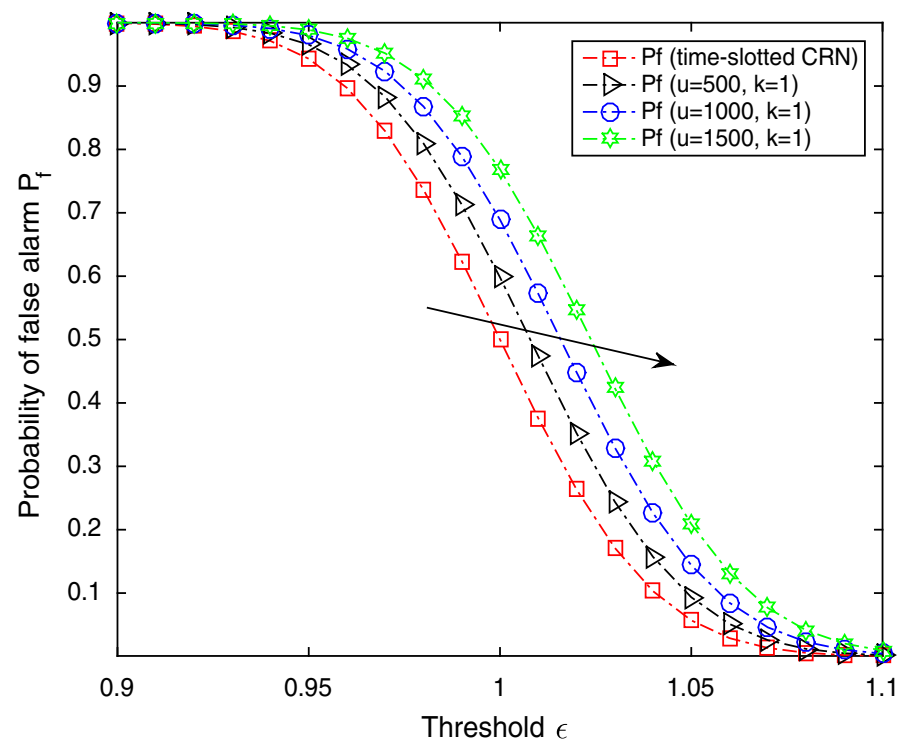

Fig. 7 Probability of false alarm versus threshold of TS-CR with HDC-SS and NTS-CR with FDC-SS without self-interference

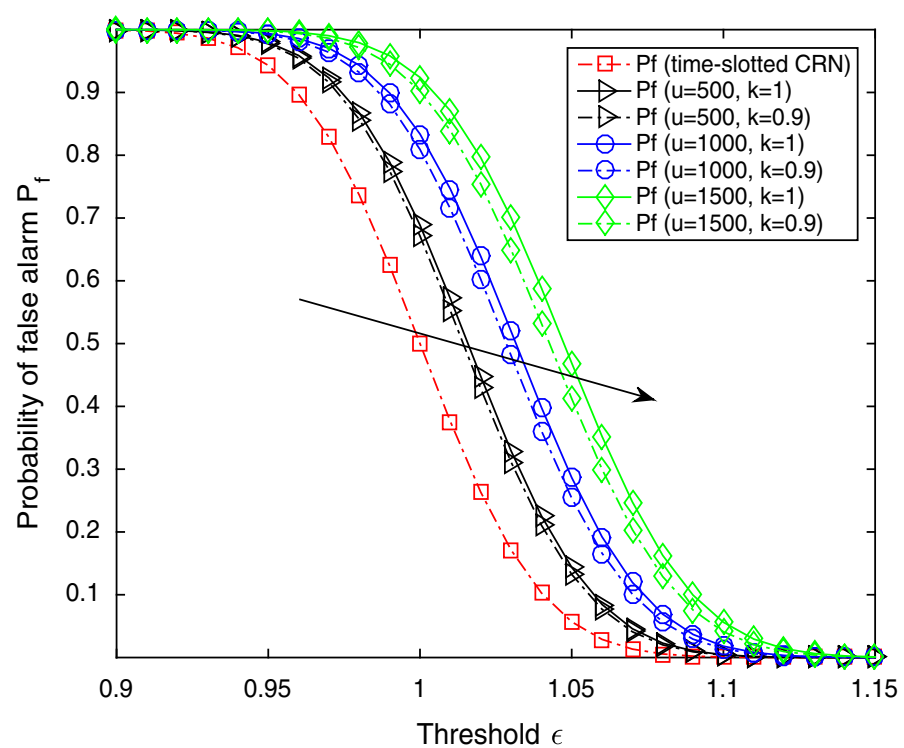

Fig. 8 Probability of false alarm versus threshold of TS-CR with HDC-SS and NTS-CR with FDC-SS with and without interference

fixed sample values let us say $u=500$ the $P_{F A}$ with interference is little higher than the $P_{F A}$ without self-interference i.e. $k=0.9$, however the performance gap is very small (Fig. 9). We can expect more degradation in $P_{F A}$ when the self-interference factor is 


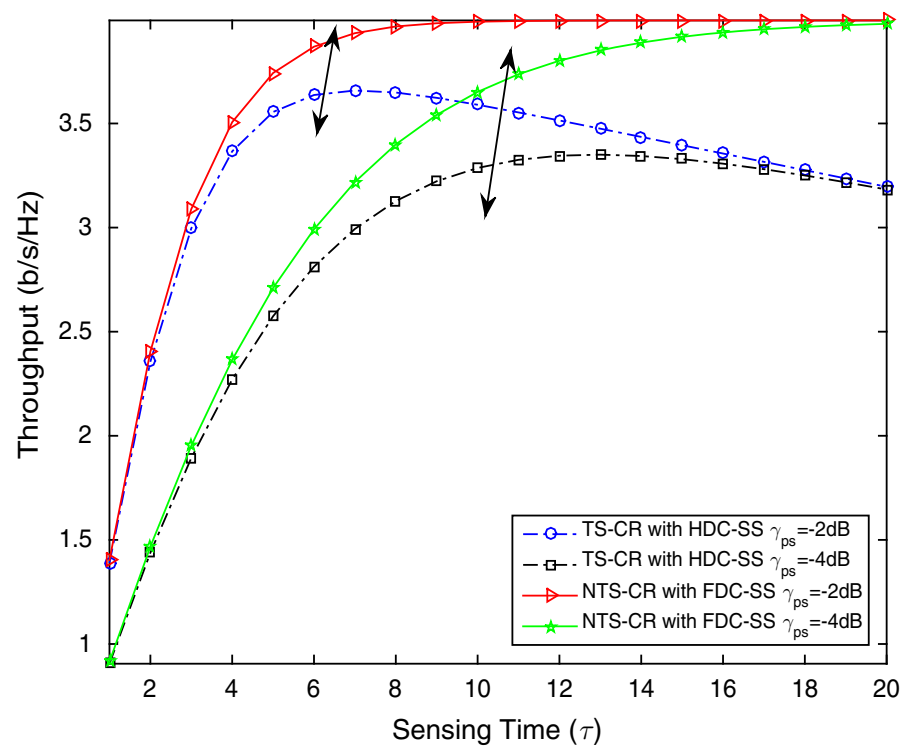

Fig. 9 Throughput versus sensing time of TS-CR with HDC-SS and NTS-CR with FDC-SS at SNR values of -2 and $-4 \mathrm{~dB}$ respectively

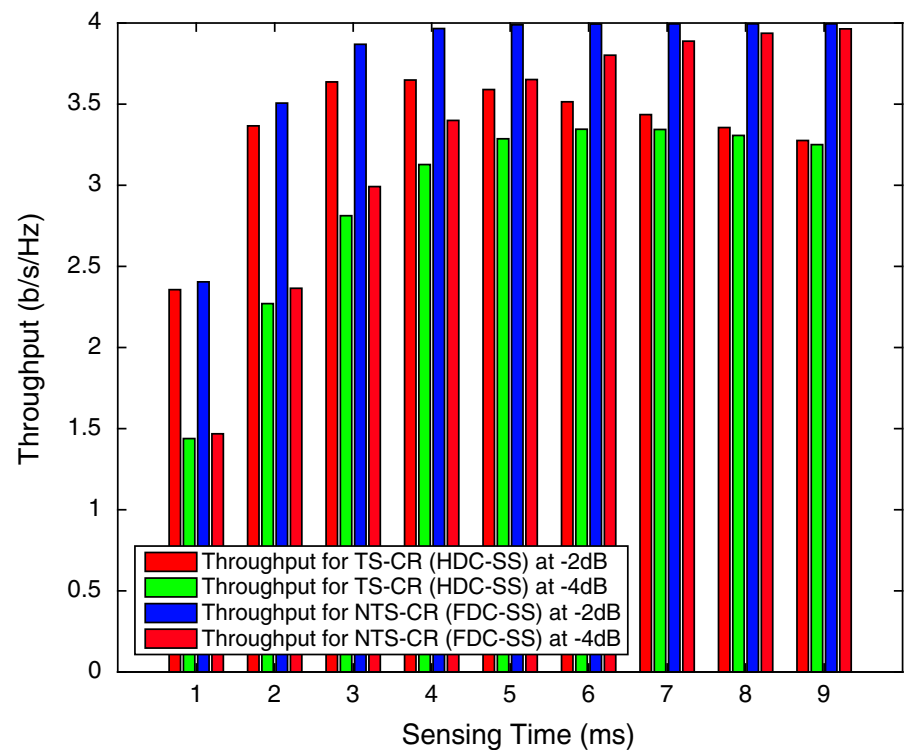

Fig. 10 Bar graph of throughput versus sensing time for TS-CR with HDC-SS and NTS-CR with FDC-SS at $\mathrm{SNR}$ values of -2 and $-4 \mathrm{~dB}$ respectively

reduced from $k=0.9$ to $k=0.1$ where sever interference can be expected. Figure 10 shows the statistical attestation of the throughput of NTS-CR which uses FDC-SS is greater than the throughput of TS-CR at different vales of SNRs. 


\section{Conclusion}

Cognitive Radio revolves around two basic and important QoS indicators which is increased throughput yield for SU and maximum protection to PU. Half-duplex communication cognitive radio which first senses and then according to sensing statistics performs data transmission. This shall reduce data transmission time for half-duplex communication cognitive radio at low SNR regimes which means throughput compromise for SU. We have seen that Full-duplex communication cognitive radio with full-duplex spectrum sensing outperforms half-duplex with half-duplex spectrum sensing schemes. We have calculated the $P_{D}, P_{F A}, P_{D}$ and throughput of the TS-CR and the NTS-CR with half and full duplex spectrum sensing. It has been proved that full-duplex cognitive radio yields more throughout than the half-duplex spectrum. We obtain approximately $5.9 \%$ increase for NTS-CR with FDC-SS at sensing time of $6 \mathrm{~ms}$. Similarly, 10\% of increase in throughput at sensing time of $10 \mathrm{~ms}$ has been observed for NTS-CR with FDC-SS for SNR values of -2 dB.

Open Access This article is distributed under the terms of the Creative Commons Attribution 4.0 International License (http://creativecommons.org/licenses/by/4.0/), which permits unrestricted use, distribution, and reproduction in any medium, provided you give appropriate credit to the original author(s) and the source, provide a link to the Creative Commons license, and indicate if changes were made.

\section{References}

1. Digham, F. F., Alouini, M.-S., \& Simon, M. K. (2007). On the energy detection of unknown signals over fading channels. IEEE Transactions on Communications, 55(1), 21-24.

2. Zhang, W., Mallik, R. K., \& Letaief, K. (2009). Optimization of cooperative spectrum sensing with energy detection in cognitive radio networks. IEEE Transactions on Wireless Communications, 8(12), 5761-5766.

3. Fuhrmann, D. R., Kelly, E. J., \& Nitzberg, R. (1992). A CFAR adaptive matched filter detector. IEEE Transactions on Aerospace and Electronic Systems, 28(1), 208-216.

4. Kapoor, S., Rao, S., \& Singh, G. (2011). Opportunistic spectrum sensing by employing matched filter in cognitive radio network. In 2011 International conference on communication systems and network technologies (CSNT) (pp. 580-583). IEEE.

5. Zeng, Y., \& Liang, Y.-C. (2007). Maximum-minimum Eigenvalue detection for cognitive radio. In IEEE 18th international symposium on personal, indoor and mobile radio communications, 2007. PIMRC 2007 (pp. 1-5). IEEE.

6. Zeng, Y., \& Liang, Y.-C. (2009). Eigenvalue-based spectrum sensing algorithms for cognitive radio. IEEE Transactions on Communications, 57(6), 1784-1793.

7. Xu, S., Zhao, Z., \& Shang, J. (2008). Spectrum sensing based on cyclostationarity. In Workshop on power electronics and intelligent transportation system, 2008. PEITS'08 (pp. 171-174). IEEE.

8. Tian, Z., \& Giannakis, G. B. (2006). A wavelet approach to wideband spectrum sensing for cognitive radios. In 1st international conference on cognitive radio oriented wireless networks and communications, 2006 (pp. 1-5). IEEE.

9. Liang, Y.-C., Zeng, Y., Peh, E. C., \& Hoang, A. T. (2008). Sensing-throughput tradeoff for cognitive radio networks. IEEE Transactions on Wireless Communications, 7(4), 1326-1337.

10. Cheng, W., Zhang, X., \& Zhang, H. (2015). Full-duplex spectrum-sensing and MAC-protocol for multichannel nontime-slotted cognitive radio networks. IEEE Journal on Selected Areas in Communications, 33(5), 820-831.

11. Sharkasi, Y. F., Ghogho, M., \& McLernon, D. (2012). Sensing-throughput tradeoff for OFDM-based cognitive radio under outage constraints. In 2012 International symposium on wireless communication systems (ISWCS) (pp. 66-70). IEEE.

12. Derakhshani, M., \& Le-Ngoc, T. (2012). Aggregate interference and capacity-outage analysis in a cognitive radio network. IEEE Transactions on Vehicular Technology, 61(1), 196-207. 
13. Chaudhari, S., Koivunen, V., \& Poor, H. V. (2009). Autocorrelation-based decentralized sequential detection of OFDM signals in cognitive radios. IEEE Transactions on Signal Processing, 57(7), 2690-2700.

14. Taherpour, A., Nasiri-Kenari, M., \& Gazor, S. (2010). Multiple antenna spectrum sensing in cognitive radios. IEEE Transactions on Wireless Communications, 9(2), 814-823.

15. Ghassemi, A., Lampe, L., Attar, A., \& Gulliver, T. A. (2010). Joint sidelobe and peak power reduction in OFDM-based cognitive radio. In 2010 IEEE 72nd vehicular technology conference fall (VTC 2010-Fall) (pp. 1-5). IEEE.

16. Ghassemi, A., \& Gulliver, T. A. (2012). PAPR reduction in OFDM based cognitive radio with blockwise-subcarrier activation. In 2012 IEEE international conference on communications (ICC) (pp. 5598-5602). IEEE.

17. Ni, C., Jiang, T., \& Peng, W. (2015). Joint PAPR reduction and sidelobe suppression using signal cancelation in NC-OFDM-based cognitive radio systems. IEEE Transactions on Vehicular Technology, 64(3), 964-972.

18. Atapattu, S., Tellambura, C., \& Jiang, H. (2011). Energy detection based cooperative spectrum sensing in cognitive radio networks. IEEE Transactions on Wireless Communications, 10(4), 1232-1241.

19. Li, X., Cao, J., Ji, Q., \& Hei, Y. (2013). Energy efficient techniques with sensing time optimization in cognitive radio networks. In 2013 IEEE wireless communications and networking conference (WCNC) (pp. 25-28). IEEE.

20. Sun, H., Nallanathan, A., Cui, S., \& Wang, C.-X. (2015). Cooperative wideband spectrum sensing over fading channels. IEEE Transactions on Vehicular Technology, 65(3), 1382-1394.

21. Chen, Y. (2010). Improved energy detector for random signals in Gaussian noise. IEEE Transactions on Wireless Communications, 9(2), 558-563.

22. Chatterjee, S., Maity, S. P., \& Acharya, T. (2014). Energy efficient cognitive radio system for joint spectrum sensing and data transmission. IEEE Journal on Emerging and Selected Topics in Circuits and Systems, 4(3), 292-300.

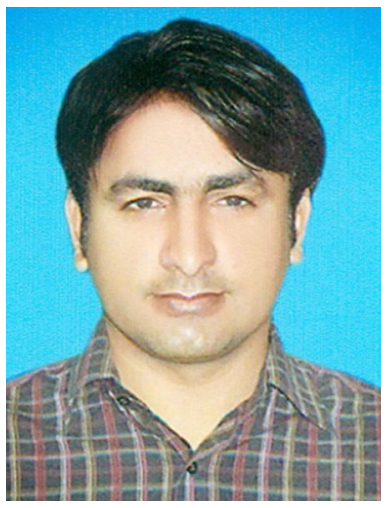

Mir Muhammad Lodro has received his B.E in Telecommunication Engineering, and M.E. in Communication Systems and Networks from Mehran UET Jamshoro in 2008 and 2014 respectively. He has also earned an MRes in Electrical and Electronic Engineering from George Green Institute for Electromagnetics Research-GGIEMR, The University of Nottingham, UK in year 2015. His research was focused on SDR and Statistical Signal Processing for Cognitive Radio in TV White Space. Additionally, he has 6 years of teaching experience at Department of Electrical Engineering, Sukkur Institute of Business Administration, Sindh, Pakistan. His areas of research interests are in broader areas of Communications, Signal Processing and Networks. $\mathrm{He}$ is member of IEEE USA and an affiliate member of IEEE Signal Processing, Communication and Networks. He is also reviewer of few international conferences and journals in the areas of CommSigNets and Antennas. He was awarded "Masters Developing Solution" scholarship and "Faculty of Engineering Research Excellence for PhD” scholarship to pursue his Master of Research (M.Res.) and Ph.D. in Electrical and Electronic Engineering from George Green Institute for Electromagnetics Research-GGIEMR, The University of Nottingham, UK. 

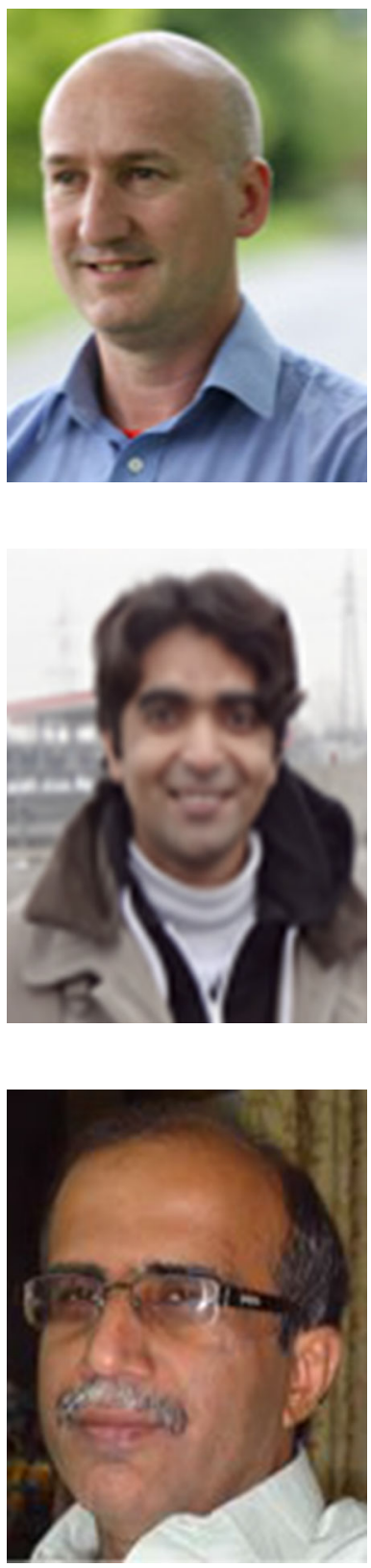

Steeve Greedy was born in Cardiff, UK in 1966. He received the MEng and Ph.D. degrees in electrical and electronic engineering from the University of Nottingham, UK, in 1998 and 2002 respectively. In 2003 he was appointed as a Senior Experimental Officer within the George Green Institute for Electromagnetics Research at the University of Nottingham, UK. His areas of research interests are computational electromagnetics and communications. He has recently focused on Cognitive Radio, SDR-based Short Range Communication Systems.

Naeem A. Mahoto received his M.E. from MUET, Pakistan. He was awarded Ph.D. scholarship under UESTPs Project for higher studies and received his Ph.D. from Politecnico Di Torino, Italy. He is currently working an Assistant Professor at MUET Pakistan. Naeem is a co-author of several research articles published in national as well as international journals. He works in the field of data mining and bioinformatics and his research interests are focused on pattern extraction, classification of electronic records in the medical domain and software engineering.

Mukhtiar A. Unar is Meritorious Professor (BPS-22) in the Department of Computer Systems Engineering and the Director of the Institute of Information \& Communication Technologies (IICT). He received the B.E in Electronic Engineering from Mehran University of Engineering and Technology, Jamshoro in 1986, the Postgraduate Diploma in Communication Engineering in 1989 from Mehran University, and the MSc and Ph.D. degrees from University of Glassgow, Scotland, UK. Dr. Mukhtiar Ali Unar is a member of IEEE (USA), an affiliate of International Federation of Automatic Control, a member of Pakistan Institute of Engineers and a member of Pakistan Engineering Council. 